\title{
Signature of helium rain and dilute cores in Jupiter's interior from empirical equations of state
}

\author{
DongDong $\mathrm{Ni}^{1,2^{*}}$ \\ 'State Key Laboratory of Lunar and Planetary Sciences, Macau University of Science and Technology, Macau 999078, China; \\ ${ }^{2}$ Institute for Planets and Exoplanets, University of California, Los Angeles, CA 90095-1567, USA
}

Key Points:

- An empirical EOS for Jupiter's interior is deduced from Juno gravity field observations in terms of the hydrostatic equation

- Comparison of the empirical EOS with Jupiter's adiabats suggests helium rain and dilute cores in Jupiter's interior

- In the planet's innermost envelope, the mass fraction of heavy elements (all elements heavier than helium) is evaluated as high as 11-13 times the solar value

Citation: Ni, D. D. (2020). Signature of helium rain and dilute cores in Jupiter's interior from empirical equations of state. Earth Planet. Phys., 4(2), 111-119. http://doi.org/10.26464/epp2020017

\begin{abstract}
Measurements of Jupiter's gravity field by Juno have been acquired with unprecedented precision, but uncertainties in the planet's hydrogen-helium equation of state (EOS) and the hydrogen-helium phase separation have meant that differences remain in the interior model predictions. We deduce an empirical EOS from Juno gravity field observations in terms of the hydrostatic equation and then investigate the structure and composition of Jupiter by comparison of the empirical EOS with Jupiter's adiabats obtained from the physical EOS. The deduced helium mass fraction suggests depletion of helium in the outermost atmosphere and helium concentration in the inner molecular hydrogen region, which is a signature of helium rain in Jupiter's interior. The deduced envelope metallicity (the heavy-element mass fraction) is as high in the innermost envelope as 11-13 times the solar value. Such a high metallicity provides sharp support to the dilute core model with the heavy elements dissolved in hydrogen and expanded outward. No matter how the core mass is varied, the empirical EOS derived from the two-layer interior model generally suggests higher densities in the innermost envelope than does the best-fit Jupiter's adiabat; this result is, again, a signature of dilute cores in Jupiter's interior. Moreover, no matter the core mass, the empirical EOS is found to exhibit an inflexion point in the deep interior, around $10 \mathrm{Mbar}$, which can be explained as the combined effect of helium concentration in the upper part and dilute cores in the lower part.
\end{abstract}

Keywords: Jupiter, interior; abundances, interiors; geophysics

\section{Introduction}

The Juno spacecraft began scientific observations of Jupiter on 27 August 2016 and has measured Jupiter's gravitational field to high precision. The accuracy of the even harmonic coefficients $J_{4}, J_{6}$, and $J_{8}$ has been improved by more than a factor of five compared with previous values based on data from Pioneer 10 and 11 and from Voyager 1 and 2 (Folkner et al., 2017; Bolton et al., 2017). The higher harmonics $J_{10}$ and $J_{12}$ have been measured through precise Doppler tracking of the Juno spacecraft (less et al., 2018). The low-degree even gravitational harmonics are affected by the shape and internal structure of Jupiter in its hydrostatic equilibrium under the effect of rotational distortion. To describe accurately the shape and internal structure of Jupiter, various interior models with new ingredients have been proposed by several authors (Guillot et al., 1997; Guillot, 1999; Hubbard, 1999; Nettelmann et al., 2012; Vazan et al., 2015; Hubbard and Militzer, 2016;

Correspondence to: D. D. Ni, ddni@must.edu.mo

Received 29 OCT 2019; Accepted 12 DEC 2019.

Accepted article online 09 JAN 2020.

C 2020 by Earth and Planetary Physics.
Kong DL et al., 2016, 2018; Debras and Chabrier, 2019; Ni DD, 2019). To date, most models of Jupiter's interior have been in the direction of forward modeling. The internal structure of Jupiter is described given an empirical or simulated EOS that describes the microscopic properties of planetary matter. But uncertainties in the hydrogen-helium EOS for Jupiter and the hydrogen-helium phase separation (Hubbard et al., 2002; Saumon and Guillot, 2004; Guillot, 2005; Fortney and Nettelmann, 2010) have led to inconsistent interior model results that differ in their predictions of Jupiter's core mass, envelope metallicity, helium concentration, and dilute cores, etc. (Miguel et al., 2016; Debras and Chabrier, 2019; Ni DD, 2019). These disagreements pose a top challenge to knowledge of the internal structure of Jupiter, since an accurate estimation of EOSs at high pressures is still an open problem; at present, laboratory measurements cannot reach the pressure of interest for Jupiter's deep interior.

Anderson and Schubert (2007) and Helled et al. (2015) proposed an alternative model for the internal rotation of Saturn, which proceeded in the direction of backward modeling. In their approach, modeling of the planet's interior does not require the hydrogenhelium EOS; instead, Saturn's EOS is inferred from the planet's de- 
duced pressure-density profile. This model is consistent with the available detecting data and interprets well the key features of Saturn. Helled et al. (2009) further investigated Saturn's interior composition and precession rate using this backward model. It has been recognized that imprecision in the hydrogen-helium EOS leads to greater uncertainties in Jupiter's interior than in the case of Saturn's interior. So it is all the more relevant to generalize the backward viewpoint and apply it to Jupiter's interior. In anticipation of Juno gravity measurements, Helled et al. (2011) used the backward model to investigate the range of possible moments of inertia (Mol), suggesting that measurements of Mol to a few tenths of a percent could provide an additional constraint on Jupiter's interior. Ni DD (2018) analyzed the dependence of Mol and tidal Love numbers $k_{2}$ on the core properties. It was found that combined measurements of $\mathrm{Mol}$ and $k_{2}$ can be used to further constrain both the core mass and size. In this contribution, we intend to infer the interior EOS from an empirical two-layer model and then investigate the distribution of helium and heavy elements in Jupiter's interior. This would yield answers to some basic questions about the structure and composition of Jupiter. In order to achieve more realistic profiles of the planet's atmosphere, in-situ density measurements from the Galileo entry probe (Galileo, 2002) are used as the outer boundary conditions instead of the zero density and density derivative at the surface.

In Section 2, we describe the empirical two-layer structure model for our calculations of the planet's radial density profile, radial pressure profile, and empirical EOS characterizing the densitypressure relationship. The gravitational harmonics are calculated using the fifth-order theory of figures; the new measurements from Juno are used to constrain the internal density profile. The model makes no assumption about the depth variation of elements. In Section 3, the physical EOSs for pure hydrogen and pure helium are presented and the additive volume rule is discussed to construct Jupiter's adiabats for mixtures of hydrogen and helium (and perhaps heavy elements). Section 4 presents the distribution of helium and heavy elements at which we have arrived by comparing the best-fit adiabat constructed from the physical EOSs with the empirical EOS. Finally, the main results of this work are summarized in Section 5.

\section{Empirical Equations of State in Jupiter's Interior}

Jupiter is made of a fluid envelope and possibly a compact central core. In the empirical two-layer structure model, the envelope density is assumed to have the form of a polynomial in radius, and the core density is approximated to be constant. The fractional internal density $\eta(x)=\rho(s)\left(4 \pi R_{\mathrm{p}}^{3} / 3 M\right)$ ( $M$ is the planet's total mass) is expressed in terms of the scaled mean radius $x=s / R_{\mathrm{p}}\left(R_{\mathrm{p}}\right.$ is the planet's mean radius) as (Ni DD, 2018)

$$
\begin{aligned}
& \eta_{\mathrm{E}}(x)=\sum_{i=0}^{5} a_{i} x^{i}, x_{\mathrm{C}} \leqslant x \leqslant 1, \\
& \eta_{\mathrm{C}}(x)=a_{\mathrm{C}}, 0 \leqslant x \leqslant x_{\mathrm{C}} .
\end{aligned}
$$

The envelope density $\eta_{\mathrm{E}}(x)$ varies monotonically with the radius $x$; the core density $\eta_{\mathrm{C}}(x)$ is never lower than $\eta_{\mathrm{E}}(x)$. The quantity $x_{\mathrm{C}}$ denotes the core-envelope boundary (CEB); the ratio of core mass to total mass is given by $m_{\mathrm{C}}=a_{\mathrm{C}} x_{\mathrm{C}}^{3}$. Contrary to previous work in which the envelope density and its derivative were set to zero at the surface $x=1$, we use in-situ density measurements from the Galileo entry probe (Galileo, 2002) to constrain the polynomial coefficients. Also, mass conservation requires that the total mass must be equal to Jupiter's known mass,

$$
\begin{aligned}
& 1-m_{\mathrm{C}}=a_{0}\left(1-x_{\mathrm{C}}^{3}\right)+\frac{3}{4} a_{1}\left(1-x_{\mathrm{C}}^{4}\right)+\frac{3}{5} a_{2}\left(1-x_{\mathrm{C}}^{5}\right) \\
& +\frac{1}{2} a_{3}\left(1-x_{\mathrm{C}}^{6}\right)+\frac{3}{7} a_{4}\left(1-x_{\mathrm{C}}^{7}\right)+\frac{3}{8} a_{5}\left(1-x_{\mathrm{C}}^{8}\right) .
\end{aligned}
$$

In combination with the above constraints, the six polynomial coefficients $a_{i}$ are determined by a nonlinear least squares fitting of the available data on Jupiter's shape and gravity field.

The gravitational zonal harmonic coefficients are weighted integrals over the internal density distribution $\rho(r, \theta)$,

$$
J_{\ell}=-\frac{1}{M R_{\mathrm{eq}}^{\ell}} \int_{V} r^{\ell} P_{\ell}(\cos \theta) \rho(r, \theta) \mathrm{d}^{3} r,
$$

where $R_{\text {eq }}$ is the equatorial radius of the planet. In practice, a reasonable guess of $\eta_{\mathrm{E}}(x)$ is provided, the even zonal harmonics $J_{2 i}$ are calculated, and differences between the calculated harmonics and the observed ones are then used to correct the polynomial coefficients. This procedure is implemented iteratively for good convergence. Here we choose to use the fifth-order theory of figures to describe Jupiter's shape, where the level surfaces are given by the figure functions $s_{2 i}(s)$ in the form (Zharkov and Trubitsyn, 1975, 1978)

$$
r(s, \theta)=s\left[1+\sum_{i=0}^{5} s_{2 i}(s) P_{2 i}(\cos \theta)\right]
$$

By definition the total potential is independent of the latitude $\theta$ at a fixed mean radius $s$. Combining the fractional internal density $\eta_{\mathrm{E}}(x)$ and the computed figure functions $s_{2 i}(s)$, and abbreviating the level surface Equation (4) as $r=s(1+\Sigma)$, one can evaluate the even zonal harmonics from Equation (3) as (Guillot et al., 2018; Ni $\mathrm{DD}, 2019)$

$J_{2 i}=-\frac{3}{2}\left(R_{\mathrm{p}} / R_{\mathrm{eq}}\right)^{2 i} \int_{-1}^{+1} \mathrm{~d} \cos \theta P_{2 i}(\cos \theta) \int_{0}^{1} \mathrm{~d} x x^{2 i+2}(1+\Sigma)^{2 i+2} \eta(x)\left(\frac{\mathrm{d} r}{\mathrm{~d} s}\right)$,

where $(\mathrm{d} r / \mathrm{d} s)$ is the derivative of $r$ with respect to $s$ obtained from Equation (4).

The internal structure of giant planets is governed by the hydrostatic equation (Anderson and Schubert, 2007)

$$
\frac{1}{\rho(s)} \frac{\mathrm{d} P(s)}{\mathrm{d} s}=-g(s)+\frac{2}{3} \omega^{2} s+\frac{s}{R_{\mathrm{p}}} g\left(R_{\mathrm{p}}\right) \varphi_{\omega}(s),
$$

where $P(s)$ is the pressure at mean radius $s, \rho(s)$ is the density at mean radius $s, g(s)=G m(s) / s^{2}$ is the gravity at mean radius $s(m(s)$ is the mass enclosed within mean radius $s), g\left(R_{\mathrm{p}}\right)$ is the surface gravity at $s=R_{\mathrm{p}}, \omega$ is the planet's angular velocity, and $\varphi_{\omega}(s)$ is a slowly varying function of the radius $s$ accounting for the dimensionless rotation perturbation at high order. Note that the term $\varphi_{\omega}(s)$ can also be evaluated in terms of the above theory of figures (Guillot and Morel, 1995). One introduces dimensionless planetary units of density, mass, and pressure, such that $\rho(s)=\eta(x)\left(3 M / 4 \pi R_{\mathrm{p}}^{3}\right), \quad m(s)=a(x) M$, and $P(s)=\xi(x)\left(3 G M^{2} / 4 \pi R_{\mathrm{p}}^{4}\right)$. The hydrostatic equation above is transformed to 


$$
\frac{1}{\eta(x)} \frac{\mathrm{d} \xi(x)}{\mathrm{d} x}=-\frac{a(x)}{x^{2}}+\left[\frac{2}{3} q_{\mathrm{rot}}+\varphi_{\omega}(x)\right] x,
$$

where $q_{\text {rot }}$ is a smallness parameter representing the ratio between centrifugal and gravitational forces, $q_{\text {rot }}=\omega^{2} R_{\mathrm{p}}^{3} / G M$. With the resulting density profile $\eta(x)$ and the calculated high-order rotation perturbation $\varphi_{\omega}(x)$, the internal pressure profile $\xi(x)$ is then obtained from the hydrostatic Equation (7) and the pressure-density profile is then inferred as an empirical EOS.

Considering that the gravitational harmonics at the planet's surface are insensitive to the precise density distribution within the innermost part of the planet (Wahl et al., 2016), we assume a constant core radius and achieve a converged core mass for Jupiter's gravity field. In our calculations, following the work of Hubbard and Militzer (2016), we choose the core radius at $x_{C}=0.15$. Three two-layer structure models with increasing core density (mass) are considered: Case (I) corresponds to the zero density jump at the CEB $\Delta \rho_{\text {CEB }}=0$, Case (II) corresponds to a core density of $\rho_{\mathrm{C}}=$ $15 \mathrm{~g} / \mathrm{cm}^{3}$, and Case (III) corresponds to a core density of $\rho_{\mathrm{C}}=$ $20 \mathrm{~g} / \mathrm{cm}^{3}$. As one proceeds from Cases (I) to (II) to (III), the core mass is increased from 6.8 to 12.1 to $16.2 M_{\oplus}$ (Earth masses). Figure 1 illustrates the density and pressure as a function of the scaled radius $x$ for the three interior models. Minor differences can be seen in the outer region $x=0.5$ to $x=1.0$; the differences become obvious in the deeper region, owing to the different core densities. Table 1 presents the calculated even zonal harmonic coefficients, comparing them with the newly observed Juno data from the PJ3 and PJ6 science orbits. It is relevant to note that the Juno harmonic coefficients contain contributions from both a rigidly rotating planet and differentially rotating zonal flows. Nevertheless, dynamical flows have minor effect on the internal density distribution of Jupiter so that the dynamical effects could be ap- proximately disregarded in this work. Static tidal Love numbers $k_{22}$ and Mol are also computed using the methods presented in $\mathrm{Ni}$ DD (2018). One can see that the calculated Love numbers $k_{22}$ are close to the Juno data within the measurement uncertainties and the calculated Mol values show good agreement with the theoretical results of Wahl et al. (2017). The empirical EOSs inferred from the three interior models are plotted in Figure 2. There are minor differences in the outer envelope, as shown in Figure 2a. In contrast to Figure $2 a$, differences can be seen in Figure $2 b$ especially for the innermost envelope. More importantly, in all three cases there is always an inflexion point of the empirical EOS in the high pressure region around $10 \mathrm{Mbar}$. This feature will be explored in Section 4.

\section{Equations of State for Pure Species and Jupiter's}

\section{Adiabats}

Hydrogen and helium form $\sim 85 \%$ of the mass of Jupiter. They are the simplest elements but exhibit remarkable properties under extreme conditions of pressure and temperature (McMahon et al., 2012). Interior models of Jupiter require the hydrogen-helium EOSs, the density as a function of temperature, pressure and composition. There are various EOSs available based on different methods and considerations (Saumon et al., 1995; Nettelmann et al., 2008, 2012; French et al., 2012; Becker et al., 2014; Militzer et al., 2008; Militzer and Hubbard, 2013). In this work we use the more recent $a b$ initio EOS REOS.3 (Becker et al., 2014) for pure hydrogen and pure helium. In the REOS.3 tables, the zero point of the specific internal energy is changed to coincide in the ideal gas regime with the SCvH EOS (Miguel et al., 2016). The resulting new EOSs are called REOS3b. If heavy elements (the $Z$ component) are considered in small quantities, they are assumed to be water
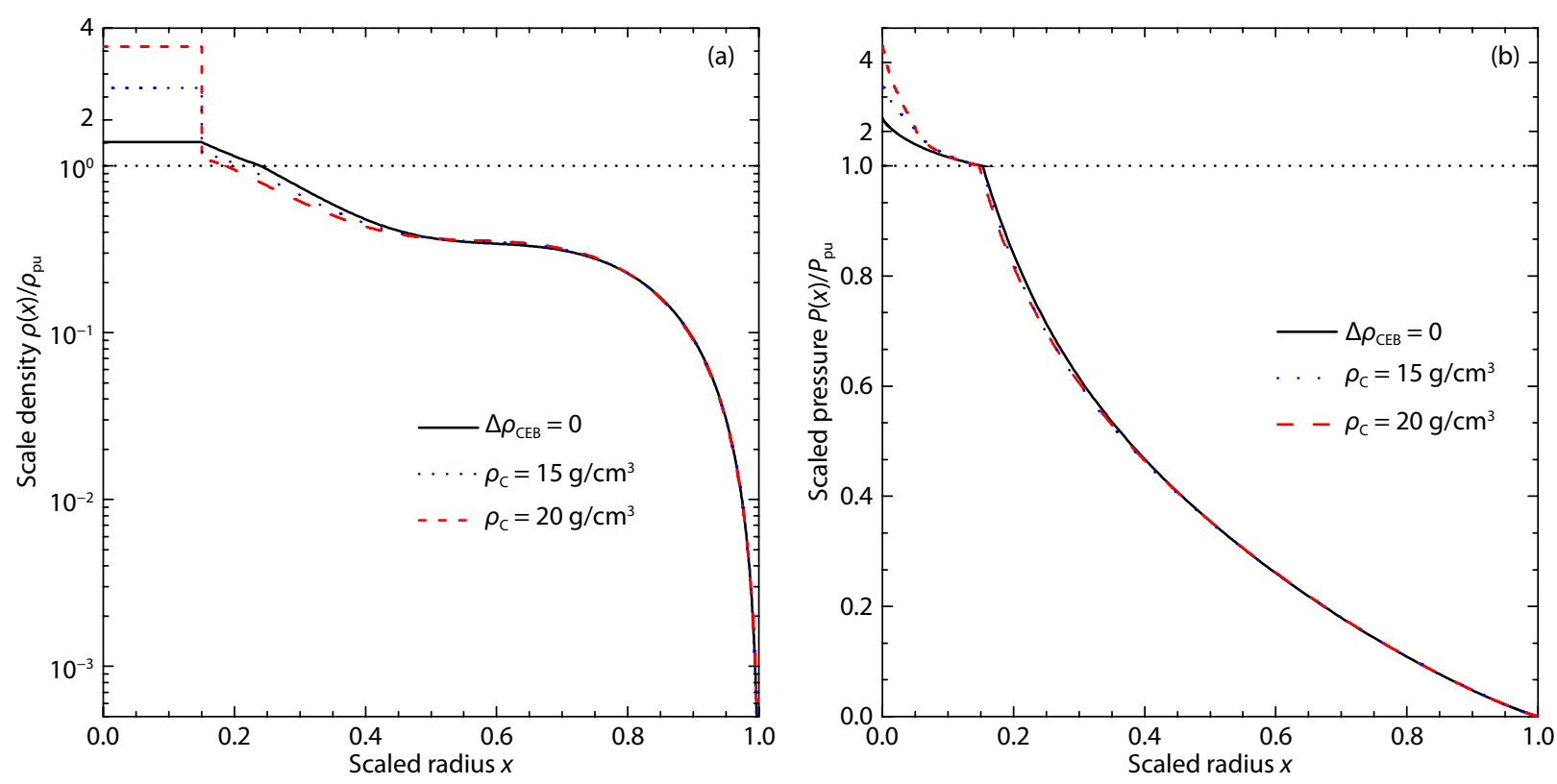

Figure 1. Profiles of density (a) and pressure (b) for three Jupiter models with the core radius $x_{C}=0.15$. Black solid, blue dotted, and red dashed curves correspond to the zero density jump at the CEB $\Delta \rho_{\text {CEB }}=0$ [Case (I)], the core density of $\rho_{\mathrm{C}}=15 \mathrm{~g} / \mathrm{cm}^{3}\left[\right.$ Case (II)], and $\rho_{\mathrm{C}}=20 \mathrm{~g} / \mathrm{cm}^{3}[$ Case (III)], respectively. For the sake of clarity, the density values are normalized by a constant density $\rho_{\mathrm{pu}}=M / R_{\mathrm{p}}^{3}=5.555 \mathrm{~g} / \mathrm{cm}^{3}$ and the pressure values are normalized by a constant pressure $P_{\mathrm{pu}}=3 G \mathrm{M}^{2} / 4 \pi R_{\mathrm{p}}^{4}=24.06 \mathrm{Mbar}$. Note that the upper and lower parts distinguished by thin dotted horizontal lines are shown in the different scales. 
Table 1. Jupiter's even zonal harmonic coefficients $J_{2 i}$, tidal Love number $k_{22}$, and normalized moment of inertia $C / M R_{\text {eq }}^{2}$ for the three interior models Cases (I)-(III), compared with the Juno data from the PJ3 and PJ6 science orbits (less et al., 2018).

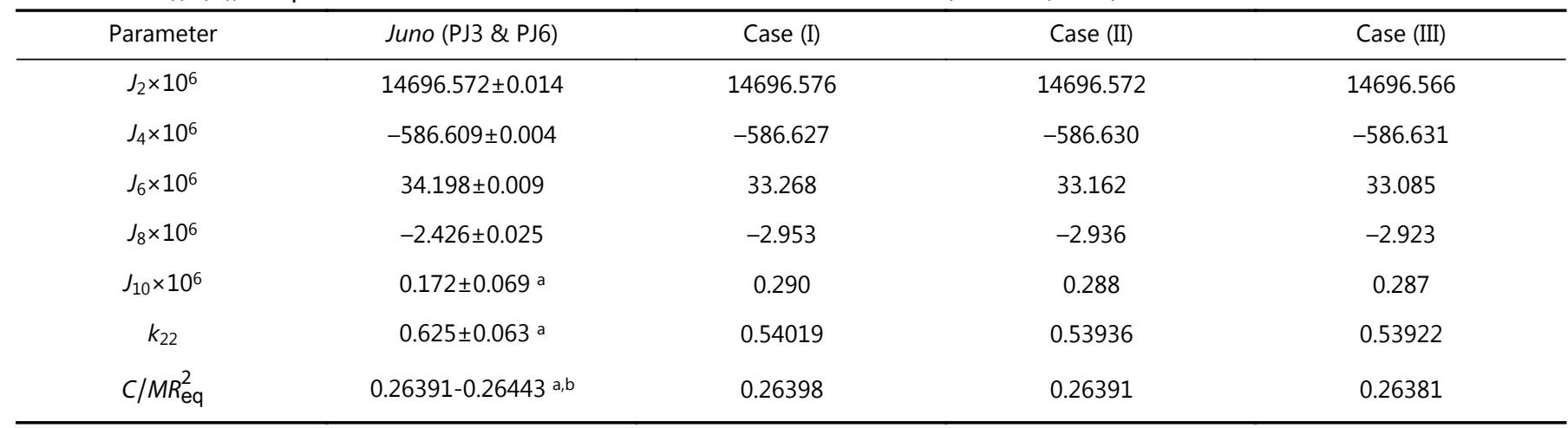

a The measured value is not used in the nonlinear least squares fitting. ${ }^{\mathrm{b}}$ The normalized moment of inertia is not measured at present; the estimations from Wahl et al. (2017) are used for reference.


Figure 2. Empirical EOSs inferred from three empirical interior models, Cases (I)-(III), with the core radius $X_{\mathrm{C}}=0.15$ : the pressure $P$ is plotted as a function of the density $\rho$ for the outer (a) and inner (b) envelopes. Black solid, blue dotted, and red dashed curves correspond to Case (I), Case (II), and Case (III), respectively.

$\left(\mathrm{H}_{2} \mathrm{O}\right)$ as a proxy for all ices. The SESAME EOS 7154 of water is used in this work (Lyon and Johnson, 1992). In the following, the mass fractions of hydrogen, helium and heavy elements are denoted by $X, Y$ and $Z(X+Y+Z=1)$, respectively.

It is of great importance to obtain an EOS for mixtures of hydrogen and helium with a minor amount of heavy elements. In principle, one can perform simulations for a mixture of hydrogen and helium (and perhaps heavy elements) with various fractions to generate the EOS database and then interpolate between these EOS to obtain an EOS for the desired composition. But this would take an enormous amount of computation time. Militzer and Hubbard (2013) provided $a b$ initio EOS data only for $N_{\mathrm{He}}=18$ helium atoms in $N_{\mathrm{H}}=220$ hydrogen atoms, corresponding to the hydrogen-helium mixture with a helium fraction of $Y /(X+Y)=0.245$. At present, the practical way is to generate the EOS for given compositions using the EOSs for pure species in terms of the additive volume rule. Extensive variables such as volume, internal energy, and entropy are strictly additive for identical systems. For ex- ample, the density for a mixture of hydrogen, helium, and heavy elements is approximated at any given pressure $P$, temperature $T$, and compositions $X / Y / Z$ as

$$
\frac{1}{\rho(P, T, Y, Z)}=\frac{X}{\rho^{\mathrm{H}}(P, T)}+\frac{Y}{\rho^{\mathrm{He}}(P, T)}+\frac{Z}{\rho^{Z}(P, T)},
$$

and the specific entropy of the $\mathrm{H}-\mathrm{He}-\mathrm{Z}$ mixture is approximated on a grid of pressures $P$, temperatures $T$, and compositions $X / Y / Z$ as

$$
S(P, T, Y, Z)=X S^{\mathrm{H}}(P, T)+Y S^{\mathrm{He}}(P, T)+Z S^{Z}(P, T)+S_{\text {mix }}(P, T, Y, Z) .
$$

To simplify the derivation we approximate the mixing entropy $S_{\text {mix }}$ by the ideal entropy of mixing of gases. If small quantities of heavy elements are not taken into account, the above two expressions (8) and (9) with $Z=0$ are for the hydrogen-helium mixture.

Jupiter is thought to have an isentropic and mostly convective envelope (Hubbard, 1968), although convection might be suppressed by compositional gradients. The envelope entropy 
$S(P, T, Y, Z)$ is determined by the measurements at the pressure of 1 bar (Saumon et al., 1995). The Galileo entry probe in Jupiter yielded the composition of $Y /(X+Y)=0.238 \pm 0.007$ and the temperature of $166 \mathrm{~K}$ at 1 bar. Once the envelope entropy $S(P, T, Y, Z)$ has been specified, Jupiter's adiabat $\rho(P, T, Y, Z)$ is constructed using the EOSs for pure species and the element abundances $X / Y / Z$. Figure 3 shows Jupiter's interior adiabats for the $\mathrm{H}-\mathrm{He}$ mixture with $Y=0.238$ and for the $\mathrm{H}-\mathrm{He}-\mathrm{Z}$ mixture with $Z \approx 0.017$ and $Y=$ $0.238(1-Z)=0.234$. Note that Jupiter's interior could depart from such a straightforward adiabatic description, because the real situation may be more complex than what we consider here. The hydrogen-helium phase separation region may bring in a jump in both temperature and entropy for the inner envelope, and there may exist a dilute core in the deep interior (Hubbard and Militzer, 2016; Wahl et al., 2017; Guillot et al., 2018; Ni DD, 2019).

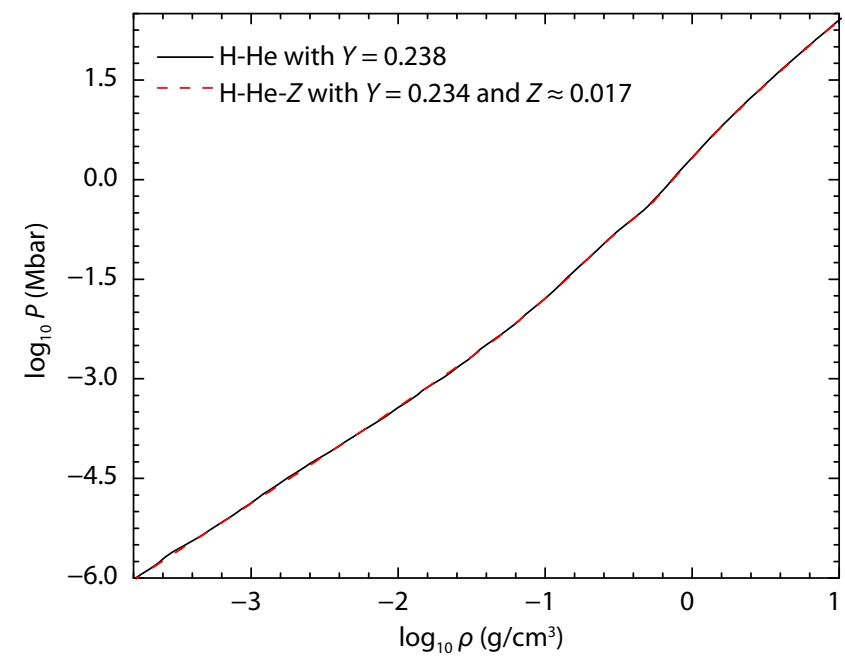

Figure 3. Jupiter's interior adiabats derived from the physical EOSs for pure species: the $\mathrm{H}-\mathrm{He}$ mixture with $Y=0.238$ (black solid curve) and the $\mathrm{H}-\mathrm{He}-\mathrm{Z}$ mixture with $Y=0.234$ and $Z \approx 0.017$ (red dashed curve).

\section{Model Results}

The empirical model of Jupiter does not directly account for the molecular-to-metallic phase transition of hydrogen or the variation with depth in the concentration of helium or the dilute core in the deep interior; nevertheless, the resulting empirical EOS is consistent with the available data on Jupiter's shape and gravity field. Therefore it appears to reflect some crucial features of Jupiter and may thus serve as a useful guide to quick assessment of Jupiter's interior. In turn, the comparison of the empirical EOS with Jupiter's adiabat can be used to deduce the relative element abundances in Jupiter's interior. As for the homogeneous hydrogen-helium mixture, the bulk abundance of helium $Y$ can be inferred by the best fit of the physical EOS models to the empirical EOS over the entire domain of pressure spanned by Jupiter's interior. Taking interior model Case (I) for an example, the resulting bulk $Y$ value is 0.306 . Figure 4 illustrates a comparison of the empirical EOS of interior model Case (I) with the best-fit adiabatic EOS from the REOS3b data. The solid curve represents the derived empirical EOS and the dashed curve represents the adiabatic EOS derived from the REOS3b data. As can be seen from the fig-

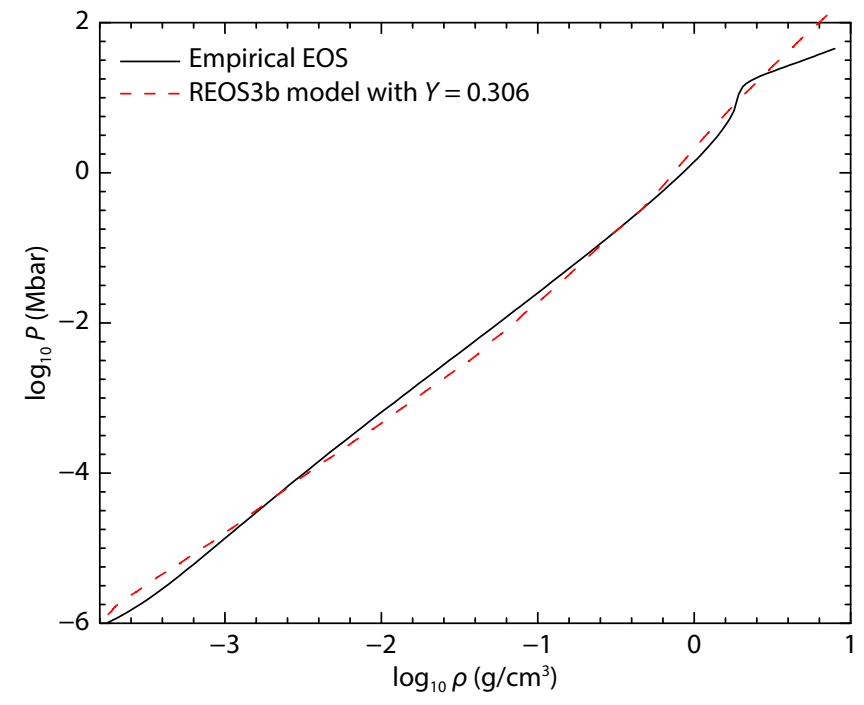

Figure 4. Comparison of the empirical EOS derived from interior model Case (I) with the best-fit adiabatic EOS obtained from REOS3b data over the entire domain of pressure spanned by Jupiter's interior.

ure, there are large differences between the empirical EOS and the adiabatic EOS. Such large deviations indicate that assuming a homogeneous hydrogen-helium mixture is insufficient to describe Jupiter's interior. The molecular-to-metallic phase transition of hydrogen, which is still not well understood either experimentally or theoretically, is expected to occur beyond the pressure of 1 Mbar for Jupiter. With these considerations in mind, Jupiter's envelope is divided into three superposed, mostly homogeneous regions: (1) the outermost atmosphere with 1 bar $\leq P<1$ kbar, (2) the inner molecular hydrogen region with $1 \mathrm{kbar} \leq P \leq 1$ Mbar, (3) the innermost region with $P>1$ Mbar and consisting of a metallic hydrogen envelope above a central dense core. The division between the latter two regions is rather indefinite, owing to either the molecular-to-metallic phase transition of hydrogen, or the hydrogen-helium separation, or both. The division between the innermost envelope and the dense central core might also be rather indistinct, since the dilute core model of Wahl et al. (2017) suggests that the heavy elements are dissolved in the innermost region and expanded outward through a region 0.3-0.5 times Jupiter's radius. In the following section we deduce the compositions of these three regions by best-fitting the empirical EOSs using the physical EOSs for pure species. We suggest that this approach has considerable potential to yield answers to some basic questions about the structure and composition of Jupiter.

In-site measurements of Jupiter's outermost atmosphere from the Galileo probe yielded the helium mass fraction of $Y /(X+Y)=0.238 \pm$ 0.007 , which is smaller than the protosolar value of $Y_{\text {proto }} /\left(X_{\text {proto }}+\right.$ $\left.Y_{\text {proto }}\right)=0.277 \pm 0.006$ (Serenelli and Basu, 2010). The measured atmospheric helium depletion has been understood in Guillot et al. (1997) and Militzer et al. (2016) as helium droplets raining down in Jupiter's atmosphere. In order to gain insight into this, we consider the hydrogen-helium mixture and analyze the distribution of helium in the envelope. To deduce the helium mass fraction $Y$, the fitting procedure described above is repeated for different interior models - Cases (I)-(III) - and for different pressure regions. The best-fit $Y$ values are summarized in Table 2 . The best-fit 
Table 2. Best-fit values of the helium mass fraction $Y$ for the hydrogen-helium mixture in different pressure regions.

\begin{tabular}{cccc}
\hline $\log _{10} P$ (Mbar) & $\begin{array}{c}-6 \text { to }-3 \\
\text { Best-fit } Y_{0}\end{array}$ & $\begin{array}{c}-3 \text { to } 0 \\
\text { Best-fit } Y_{1}\end{array}$ & $\begin{array}{c}>0 \\
\text { Best-fit } Y_{2}\end{array}$ \\
\hline Case (I) & $0.258 \pm 0.047$ & $0.437 \pm 0.078$ & $0.416 \pm 0.084$ \\
Case (II) & $0.262 \pm 0.046$ & $0.436 \pm 0.079$ & $0.401 \pm 0.085$ \\
Case (III) & $0.263 \pm 0.046$ & $0.438 \pm 0.081$ & $0.393 \pm 0.085$ \\
\hline
\end{tabular}

$Y$ values from all three interior models are slightly larger than the Galileo probe value for the outermost atmosphere, which can be easily understood since small amounts of heavy elements are not yet taken into account. More importantly, the best-fit $Y$ values are significantly increased by more than $66 \%$ from the outmost atmosphere to the inner molecular hydrogen region. This clearly demonstrates that there is helium depletion in the outermost atmosphere and helium concentration in the inner molecular hydrogen region. One can also notice that the three interior models Cases (I)-(III) yield similar results in the outer two regions. This is not so surprising since, as demonstrated in Figure $2 \mathrm{a}$, there are minor differences in the empirical EOSs of the outer two regions. The differences of the helium mass fraction emerge in the innermost region. In general, the deduced helium mass fraction $Y_{2}$ shows a decreasing tendency with increasing core mass from Cases (I) to (II) to (III). The best-fit $Y_{2}$ values are all larger than the protosolar value, suggesting the existence of heavy elements $Z$. And the gradual decrease of $Y_{2}$ indicates that two-layer structure models of Jupiter with heavy cores exhibit a smaller amount of heavy elements mixed in the metallic hydrogen envelope, which is consistent with the available interior models that use different EOSs as input parameters (Guillot, 1999; Saumon and Guillot, 2004).

The comparison of the $P-\rho$ profiles between the empirical and physical models is illustrated in Figure 5 for interior models Cases (I)-(III). It can be seen that the empirical EOSs derived from Cases (I)-(III) produce similarly good fits. With respect to the global fit over Jupiter's entire envelope (as shown in Figure 4), interior model Case (I) demonstrates better agreement with the empirical EOS specifically in the middle region. It is known that the pressuredensity relationship in planetary interiors is affected by several factors such as temperature and composition and that such effects are expected to be particularly profound in the deeper region due to large uncertainties of these factors. So there are relatively large deviations of the best-fit adiabats from the empirical EOSs in the high pressure region. In the innermost envelope with high pressure $P>20 \mathrm{Mbar}$, one can see that the densities obtained from the REOS3b model are generally lower than the one obtained from the empirical EOS for all interior models Cases (I)-(III). This feature can also be found in the result of Helled et al. (2009) for Saturn, where the density difference $\Delta \log _{10} \rho=\left(\log _{10} \rho-\right.$ $\log _{10} \rho_{\text {empirical }}$ ) becomes negative and gets enhanced with increasing pressure in the deeper envelope. This implies rather different composition above the core than in the outer envelope, which could be understood as the increased presence of heavy elements in the deeper envelope.

The dilute core model of Wahl et al. (2017) demonstrated that heavy elements are dissolved in the hydrogen-helium mixture and expanded outward through a portion of Jupiter. The change in composition would not only affect the EOS in the metallic envelope but also bring in the higher density above the core. On the other hand, the preliminary Jupiter model of Hubbard and Militzer (2016) suggested that hydrogen-helium immiscibility reduces the heat transport in the region around $P \geq 2 \mathrm{Mbar}$ and hence brings in a higher-entropy adiabat for interior pressures larger than 1 Mbar. The higher-entropy adiabat means higher temperatures and lower densities at pressures of interest beneath the hydrogen-helium immiscibility region. These two effects on the interior densities are not taken into account in constructing Jupiter's adiabats. As a result, the inflexion point of the empirical pressure-density profile is not reproduced by the best-fit adiabats. This can be regarded as indirect support for the existence of helium rain and dilute cores.

Next, we introduce a minor amount of water (representative of heavy elements) into the hydrogen-helium mixture and analyze the interior compositions. First, the mass fractions of helium and heavy elements, $Y$ and $Z$, are determined by best-fitting the empirical EOS using the physical EOSs for pure species, as described above. We find that the hydrogen mass fraction remains almost the same but the partial amounts of helium are replaced by water. This counter-balance correlation between $Y$ and $Z$ makes it difficult to determine explicitly the best-fit values of $Y$ and $Z$ in the three different pressure regions. An alternative way of addressing heavy elements is needed. For interior models Cases (I)-(III), as demonstrated in Section 2, there are minor differences among the empirical EOSs in the outer two regions with $P \leq 1 \mathrm{Mbar}$, and the inner envelope between $P=1 \mathrm{Mbar}$ and the CEB spans over the total interior of $x=0.150$ to $x=0.856$ and exhibits about 0.875 of the total mass. Therefore, special attention needs to be paid to the inner envelope with $P>1 \mathrm{Mbar}$. It is well known that the total helium to hydrogen ratio in Jupiter should be equal to its value in the protosolar nebula $Y_{\text {proto }} /\left(X_{\text {proto }}+Y_{\text {proto }}\right)=0.277 \pm 0.006$ (Serenelli and Basu, 2010). Here, the helium to hydrogen ratio in the inner envelope is approximated as the protosolar value and the mass faction of heavy elements $Z$ is determined by best fitting the empirical EOSs. The best-fit values of $Z$ are summarized in Table 3. As can be seen, the deduced $Z$ values are found to be roughly 11-13 times the solar value of 0.0149 (Lodders, 2003). Such a high metallicity strongly supports the dilute core model, with heavy elements dissolved in hydrogen and expanded outward (Wahl et al., 2017). In addition, the mass of heavy elements mixed in the envelope is calculated as

$$
M_{Z}=Z M\left(a_{1}-a_{2}\right)
$$

where $a_{1}\left(a_{2}\right)$ is the mass fraction at the outer (inner) boundary of the envelope concerned. The global mass fraction of heavy elements in Jupiter is approximated by the expression $Z_{\text {global }}=\left(M_{\text {core }}+\right.$ $\left.M_{Z}\right) / M$. The core mass fraction and the global mass fraction of heavy elements are also listed in Table 3 for the three interior models Cases (I)-(III). It seems that a heavy central core corresponds to a smaller amount of heavy elements mixed into the inner envelope, and the global mass fraction of heavy elements shows small variations for interior models Cases (I-III). It is relevant to note that the best-fit adiabatic EOSs exhibit rather large devi- 


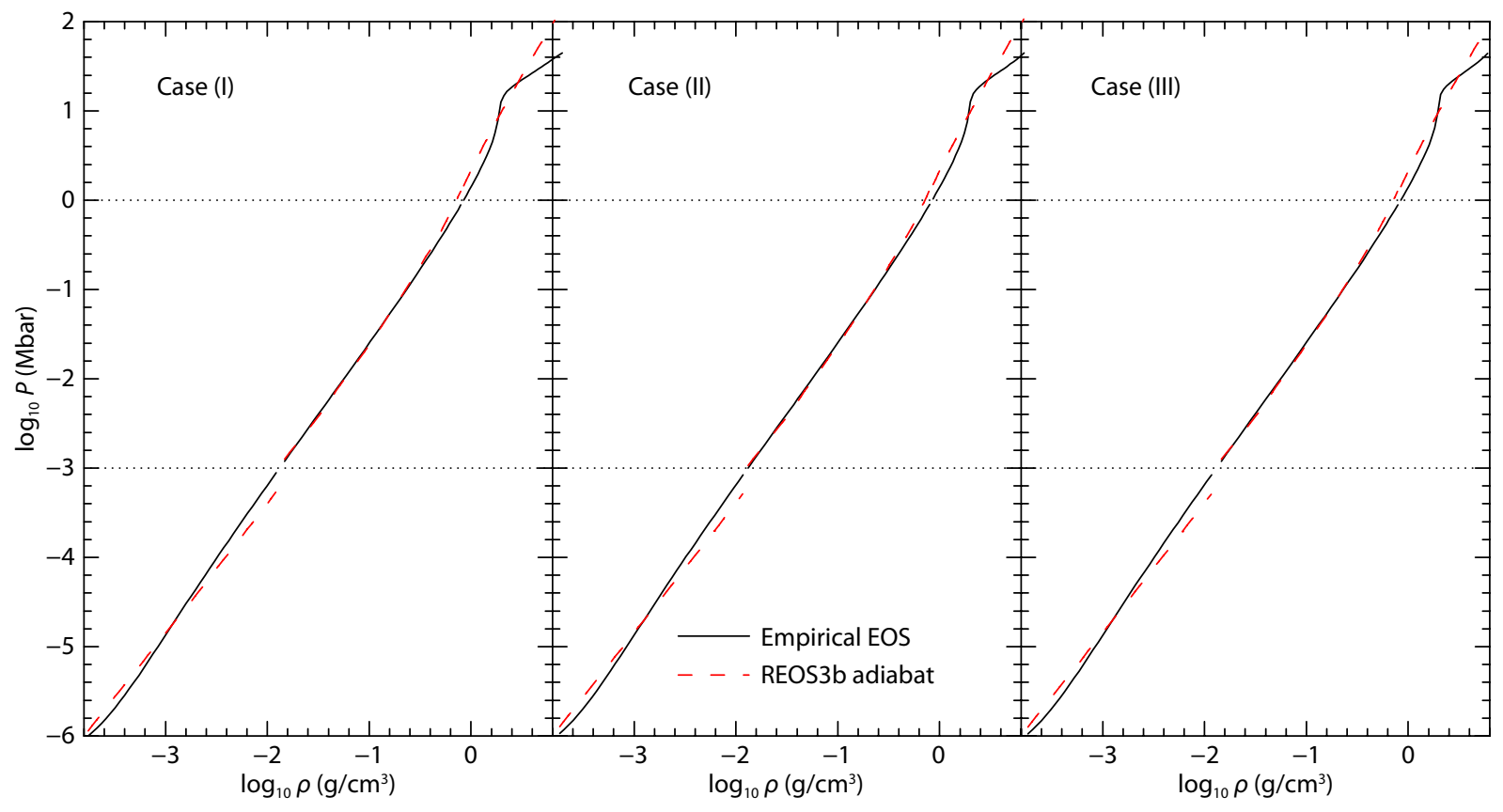

Figure 5. Comparison of the empirical EOS with the best-fit adiabatic EOS obtained from the REOS3b model in the three pressure regions separated by the horizontal dotted lines: Case (I) with the zero density jump $\Delta \rho_{\text {CEB }}=0$ (left panel), Case (II) with the core density $\rho_{C}=15 \mathrm{~g} / \mathrm{cm}^{3}$ (middle panel), and Case (III) with the core density $\rho_{\mathrm{C}}=20 \mathrm{~g} / \mathrm{cm}^{3}$ (right panel).

Table 3. Best-fit values of the mass fractions of heavy elements $Z$ for the $\mathrm{H}-\mathrm{He}-\mathrm{Z}$ mixture in the inner pressure region where $P>1 \mathrm{Mbar}$. As additional information, the core mass fraction and the global mass fraction of heavy elements are also listed for the three interior models Cases (I)-(III).

\begin{tabular}{cccc}
\hline$P>1$ Mbar & Case (I) & Case (II) & Case (III) \\
\hline Best-fit $Z$ & 0.188 & 0.172 & 0.163 \\
Core mass fraction & 0.021 & 0.038 & 0.051 \\
$Z_{\text {global }}$ & 0.189 & 0.188 & 0.191 \\
\hline
\end{tabular}

ations from the empirical EOSs in the innermost region, thus causing large uncertainties in the deduced mass of heavy elements. Furthermore, little knowledge of the EOS for heavy elements in Jupiter directly affects the deduced heavy-element mass fraction. Thus our work here provides just a rough estimation of Jupiter's heavy element abundance.

\section{Conclusion}

We have presented in this paper three empirical EOSs for Jupiter's interior, based on the two-layer structure model and consistent with the Juno gravity measurements. Jupiter's adiabats are also constructed in terms of the additive volume rule using the physical EOSs for pure species with the aid of data from the Galileo entry probe into Jupiter. The composition and structure of Jupiter are explored by comparing Jupiter's adiabats, obtained from the physical EOSs, with the empirical EOSs. For the hydrogen-helium mixture, the deduced $Y$ value is found to be increased by more than $66 \%$ from the outermost atmosphere to the inner molecular hydrogen region, as shown in Table 2 . This suggests that helium droplets fall down from the outermost atmosphere and concentrate in the inner molecular hydrogen region. Next, in addition to the hydrogen-helium mixture, the small amount of heavy elements (which are assumed to be water) is taken into account. We approximate the helium to hydrogen ratio in the inner envelope as the protosolar value $Y_{\text {proto }} /\left(X_{\text {proto }}+Y_{\text {proto }}\right)=0.277 \pm 0.006$ and adjust the envelope metallicity $Z$. The deduced $Z$ values are found to be as high as $11-13$ times the solar value of 0.0149 . This gives strong support to the dilute core model with heavy elements dissolved in hydrogen and expanded outward. One can also notice in Figure 5 that the empirical EOSs exhibit higher densities than the best-fit Jupiter's adiabat for one given pressure in the innermost envelope, and this feature becomes more evident with increasing pressure. A similar situation can be found in the work of Helled et al. (2009) for Saturn. This implies rather different composition above the central core than that of the outer envelope, which could be a signature of dilute cores. Moreover, in all three empirical EOSs, an inflexion point emerges at the high pressure region around $10 \mathrm{Mbar}$, which can be understood as evidence of heavy element dissolution in the metallic envelope and hydrogen-helium immiscibility around $P \sim 2$ Mbar. This indirectly confirms the existence of heavy element dissolution in the metallic envelope and hydrogen-helium immiscibility.

Our empirical EOSs are merely preliminary and intended for use as a reference for comparison with Jupiter's adiabats obtained from the physical EOS models. The data available on Jupiter's shape and gravity field might not be sufficient to infer realistic EOSs for Jupiter's interior. Future, new, and more abundant gravity field data would provide more accurate information on Jupiter's interior. Also, better knowledge of the EOSs for pure species, from both experimental and theoretical sides, would improve our understanding of the composition and structures of planets such as Jupiter. Besides, convection might be suppressed in some regions 
of Jupiter's interior owing to compositional gradients. The Galileo entropy, which was determined in terms of the entry probe into the outmost atmosphere, could be different from the entropy of the deep interior. It has been suggested in Hubbard and Militzer (2016) and Debras and Chabrier (2019) that hydrogen-helium immiscibility brings in a higher-entropy adiabat for interior pressures larger than 1 Mbar. The calculation of Jupiter's adiabat will be improved by using different entropies for the two envelopes. A reasonable treatment would be required to evaluate the entropy jump and the interface between these two adiabats.

\section{Acknowledgment}

We thank Y. Miguel for valuable discussions and for providing data regarding REOS3b. We also thank T. Guillot for valuable comments. This work is supported by the Science and Technology Development Fund, Macau SAR (File No. 0005/2019/A1) and the National Natural Science Foundation of China Grant No. 11761161001.

\section{References}

Anderson, J. D., and Schubert, G. (2007). Saturn's gravitational field, internal rotation, and interior structure. Science, 317(5843), 1384-1387. https://doi.org/10.1126/science.1144835

Becker, A., Lorenzen, W., Fortney, J. J., Nettelmann, N., Schöttler, M., and Redmer, R. (2014). Ab initio equations of state for hydrogen (H-REOS.3) and helium (He-REOS.3) and their implications for the interior of brown dwarfs. Astrophys. J. Suppl. Ser., 215(2), 21. https://doi.org/10.1088/00670049/215/2/21

Bolton, S. J., Adriani, A., Adumitroaie, V., Allison, M., Anderson, J., Atreya, S., Bloxham, J., Brown, S., Connerney, P. J. E., ... Wilson, R. (2017). Jupiter's interior and deep atmosphere: The initial pole-to-pole passes with the Juno spacecraft. Science, 356(6340), 821-825. https://doi.org/10.1126/science.aal2108

Debras, F., and Chabrier, G. (2019). New models of Jupiter in the context of Juno and Galileo. Astrophys. J., 872(1), 100. https://doi.org/10.3847/15384357/aaf99f

Folkner, W. M., less, L., Anderson, J. D., Asmar, S. W., Buccino, D. R., Durante, D., Feldman, M., Gomez Casajus, L., Gregnanin, M., ... Levin, S. M. (2017). Jupiter gravity field estimated from the first two Juno orbits. Geophys. Res. Lett., 44(10), 4694-4700. https://doi.org/10.1002/2017GL073140

Fortney, J. J., and Nettelmann, N. (2010). The interior structure, composition, and evolution of giant planets. Space Sci. Rev., 152(1-4), 423-447. https://doi.org/10.1007/s11214-009-9582-x

French, M., Becker, A., Lorenzen, W., Nettelmann, N., Bethkenhagen, M., Wicht, J., and Redmer, R. (2012). Ab initio simulations for material properties along the Jupiter adiabat. Astrophys. J. Suppl. Ser., 202(1), 5. https://doi.org/10.1088/0067-0049/202/1/5

Galileo probe archive. (2002). Available at https://pdsatmospheres.nmsu.edu/cgi-bin/getdir.pl?dir=index $\backslash \&$ volume=gp $\_0001$

Guillot, T., and Morel P. (1995). CEPAM: a code for modeling the interiors of giant planets. Astron. Astrophys. Suppl. Ser., 109, 109-123. https://doi.org/10.1002/asna.2103160610

Guillot, T., Gautier, D., and Hubbard, W. B. (1997). New constraints on the composition of Jupiter from Galileo measurements and interior models. Icarus, 130(2), 534-539. https://doi.org/10.1006/icar.1997.5812

Guillot, T. (1999). A comparison of the interiors of Jupiter and Saturn. Planet. Space Sci., 47(10-11), 1183-1200. https://doi.org/10.1016/S00320633(99)00043-4

Guillot, T. (2005). The interiors of giant planets: Models and outstanding questions. Annu. Rev. Earth Planet. Sci., 33(1), 493-530. https://doi.org/10.1146/annurev.earth.32.101802.120325

Guillot, T., Miguel, Y., Militzer, B., Hubbard, W. B., Kaspi, Y., Galanti, E., Cao, H.,
Helled, R., Wahl, S. M., ... Bolton, S. J. (2018). A suppression of differential rotation in Jupiter's deep interior. Nature, 555(7695), 227-230. https://doi.org/10.1038/nature25775

Helled, R., Schubert, G., and Anderson, J. D. (2009). Empirical models of pressure and density in Saturn's interior: implications for the helium concentration, its depth dependence, and Saturn's precession rate. Icarus, 199(2), 368-377. https://doi.org/10.1016/j.icarus.2008.10.005

Helled, R., Anderson, J. D., Schubert, G., and Stevenson, D. J. (2011). Jupiter's moment of inertia: a possible determination by Juno. Icarus, 216(2), 440-448. https://doi.org/10.1016/j.icarus.2011.09.016

Helled, R., Galanti, E., and Kaspi, Y. (2015). Saturn's fast spin determined from its gravitational field and oblateness. Nature, 520(7546), 202-204. https://doi.org/10.1038/nature14278

Hubbard, W. B. (1968). Thermal structure of Jupiter. Astrophys. J., 152, 745-754. https://doi.org/10.1086/149591

Hubbard, W. B. (1999). Gravitational signature of Jupiter's deep zonal flows. Icarus, 137(2), 357-359. https://doi.org/10.1006/icar.1998.6064

Hubbard, W. B., Burrows, A., and Lunine, J. I. (2002). Theory of giant planets. Annu. Rev. Astron. Astrophys., 40, 103-136. https://doi.org/10.1146/annurev.astro.40.060401.093917

Hubbard, W. B., and Militzer, B. (2016). A preliminary Jupiter model. Astrophys. J., 820(1), 80. https://doi.org/10.3847/0004-637X/820/1/80

less, L., Folkner, W. M., Durante, D., Parisi, M., Kaspi, Y., Galanti, E., Guillot, T., Hubbard, W. B., Stevenson, D. J., ... Bolton, S. J. (2018). Measurement of Jupiter's asymmetric gravity field. Nature, 555(7695), 220-222. https://doi.org/10.1038/nature25776

Kong, D. L., Zhang, K. K., and Schubert, G. (2016). A fully self-consistent multilayered model of Jupiter. Astrophys. J., 826(2), 127. https://doi.org/10.3847/0004-637X/826/2/127

Kong, D. L., Zhang, K. K., Schubert, G., and Anderson, J. D. (2018). Origin of Jupiter's cloud-level zonal winds remains a puzzle even after Juno. Proc NatI Acad Sci USA, 115(34), 8499-8504. https://doi.org/10.1073/pnas.1805927115

Lodders, K. (2003). Solar system abundances and condensation temperatures of the elements. Astrophys. J., 591(2), 1220-1247. https://doi.org/10.1086/375492

Lyon, S. P., and Johnson, J. D. (1992). LANL Rep. LA-UR-92-3407 (Los Alamos: LANL).

McMahon, J. M., Morales, M. A., Pierleoni, C., and Ceperley, D. M. (2012). The properties of hydrogen and helium under extreme conditions. Rev. Mod. Phys., 84(4), 1607-1653. https://doi.org/10.1103/RevModPhys.84.1607

Miguel, Y., Guillot, T., and Fayon, L. (2016). Jupiter internal structure: the effect of different equations of state. $A \& A, 596, A 114$. https://doi.org/10.1051/0004-6361/201629732

Militzer, B., Hubbard, W. B., Vorberger, J., Tamblyn, I., and Bonev, S. A. (2008). A massive core in Jupiter predicted from first-principles simulations. Astrophys. J. Lett., 688(1), L45. https://doi.org/10.1086/594364

Militzer, B., and Hubbard, W. B. (2013). Ab initio equation of state for hydrogenhelium mixtures with recalibration of the giant-planet mass-radius relation. Astrophys. J., 774(2), 148. https://doi.org/10.1088/0004-637X/774/2/148

Militzer, B., Soubiran, F., Wahl, S. M., and Hubbard, W. (2016). Understanding Jupiter's interior. J. Geophys. Res. Planets, 121(9), 1552-1572. https://doi.org/10.1002/2016JE005080

Nettelmann, N., Holst, B., Kietzmann, A., French, M., Redmer, R., and Blaschke, D. (2008). Ab initio equation of state data for hydrogen, helium, and water and the internal structure of Jupiter. Astrophys. J., 683(2), 1217-1228. https://doi.org/10.1086/589806

Nettelmann, N., Becker, A., Holst, B., and Redmer, R. (2012). Jupiter models with improved $A b$ initio hydrogen equation of state (H-REOS.2). Astrophys. J., 750(1), 52. https://doi.org/10.1088/0004-637X/750/1/52

$\mathrm{Ni}$, D. D. (2018). Empirical models of Jupiter's interior from Juno data: moment of inertia and tidal Love number $k_{2}$. A\&A, 613, A32. https://doi.org/10.1051/0004-6361/201732183

$\mathrm{Ni}, \mathrm{D}$. D. (2019). Understanding Jupiter's deep interior: the effect of a dilute core. A\&A, 632, A76. https://doi.org/10.1051/0004-6361/201935938

Saumon, D., Chabrier, G., and van Horn, H. M. (1995). An equation of state for 
low-mass stars and giant planets. Astrophys. J. Suppl. Ser., 99, 713-741. https://doi.org/10.1086/192204

Saumon, D., and Guillot, T. (2004). Shock compression of deuterium and the interiors of Jupiter and Saturn. Astrophys. J., 609(2), 1170-1180. https://doi.org/10.1086/421257

Serenelli, A. M., and Basu, S. (2010). Determining the initial helium abundance of the sun. Astrophys. J., 719(1), 865-872. https://doi.org/10.1088/0004$637 X / 719 / 1 / 865$

Vazan, A., Helled, R., Kovetz, A., and Podolak, M. (2015). Convection and mixing in giant planet evolution. Astrophys. J., 803(1), 32. https://doi.org/10.1088/0004-637X/803/1/32

Wahl, S. M., Hubbard, W. B., and Militzer, B. (2016). Tidal response of preliminary
Jupiter model. Astrophys. J., 831(1), 14. https://doi.org/10.3847/0004$637 \mathrm{X} / 831 / 1 / 14$

Wahl, S. M., Hubbard, W. B., Militzer, B., Guillot, T., Miguel, Y., Movshovitz, N., Kaspi, Y., Helled, R., Reese, D., ... Bolton, S. J. (2017). Comparing Jupiter interior structure models to Juno gravity measurements and the role of a dilute core. Geophys. Res. Lett., 44(10), 4649-4659. https://doi.org/10.1002/2017GL073160

Zharkov, V. N., and Trubitsyn, V. P. (1975). Fifth-approximation system of equations for the theory of figure. Soviet Astron., 19, 366.

Zharkov, V. N., and Trubitsyn, V. P. (1978). Physics of Planetary Interiors. Tucson: Pachart Pub. House. 